\title{
INVESTIGAÇÃO EXPERIMENTAL DE TRAÇOS PARA BLOCOS DE CONCRETO PARA ALVENARIA DE VEDAÇÃO COM ADIÇÃO DE RESÍDUOS DE PNEUS RECICLADOS
}

\author{
Eylisson A. Santos ${ }^{1}$, Edilberto Vitorino Borja ${ }^{2}$ \\ ${ }^{1}$ Aluno de graduação do Curso de Tecnologia em Materiais - CEFET/RN (Iniciação \\ Científica); eylisson@yahoo.com.br \\ ${ }^{2}$ Professor dos Cursos de Tecnologia em Produção da Construção Civil e Tecnologia em \\ Materiais -CEFET/RN; edilberto@cefetrn.br
}

Recebido em outubro de 2004 e Aceito em abril de 2005

\begin{abstract}
RESUMO
Em acordo com a tendência mundial de se construir com resíduos sólidos, combinando o uso de energias renováveis com a redução dos materiais inservíveis é que o presente trabalho foi desenvolvido, cujo principal objetivo é a utilização de resíduos de pneus reciclados em blocos de concreto para alvenaria de vedação, buscando desta forma, mais uma contribuição para tal fim. Com este objetivo em mente, iniciou-se, nesta etapa da pesquisa, estudos experimentais em corpos de prova de concreto em 3 (três) diferentes traços para análise inicial do comportamento deste novo material com relação a sua resistência à compressão e assim, obter a melhor composição da mistura visando sua futura utilização em blocos vazados de concreto com resíduo de pneu. Para os três proporções iniciais, o agregado graúdo foi substituído parcialmente pela borracha com porcentagens variando entre 5, 10 e 15\%, em massa. A influência da relação água/cimento na resistência à compressão do concreto sem e com resíduo de borracha também foi objeto do estudo, cujas relações variaram de 0,55, 0,73 e 0,91 para cada tipo de traço com as diferentes porcentagens de borracha acima citadas. Os resultados dos estudos experimentais são apresentados e comparados graficamente entre si.
\end{abstract}

Palavras-chaves: resíduos de pneu; concretos com resíduos; traços para blocos; reciclagem

\section{EXPERIMENTAL INVESTIGATION OF CONCRETE BLOCKS TRACES FOR SEALING MASONRY WITH ADDITION OF RECYCLED TIRES RESIDUES}

\begin{abstract}
This work has been developed according to the world tendency of building with solid residues, combining the use of energy renewable sources of energy with the reduction of the useless materials its main objective is the use of recycled tire residues in concrete blocks for sealing masonry, aiming at providing one more contribution to such purpose. With this objective in mind, in this stage of research, experimental studies in concrete test specimens were started in three different mixes for initial analysis of the behavior of this new material with regard to its compressive strength in order to get the better composition of the mixture for future application in concrete blocks. For the three initial ratios, the aggregate was partially by rubber with percentages ranging from 5, 10 and 15\%, in mass. The influence of the relation water/cement on the compressive strength of the concrete
\end{abstract}


without and with rubber residue was also object of study, whose relations varied from 0.55 , 0.73 and 0.91 for each type of mix with the different rubber percentages above mentioned. The results of the experimental studies are presented and graphically between itself.

Key words: residues of tires; concrete with residues; mixes for blocks; recycling; 


\section{INVESTIGAÇÃO EXPERIMENTAL DE TRAÇOS PARA BLOCOS DE CONCRETO PARA ALVENARIA DE VEDAÇÃO COM ADIÇÃO DE RESÍDUOS DE PNEUS RECICLADOS}

\section{INTRODUÇÃO}

Um dos principais desafios ambientais enfrentados ao redor do mundo é a disposição de pneus inservíveis em terrenos baldios, rios e lagoas, formando um grande passivo ambiental. Várias pesquisas vêm sendo desenvolvidas no âmbito de sua reutilização com objetivo de minimizá-lo.

A utilização de pneus em obras de construção civil e em muitas outras áreas, além de dar uma destinação nobre a um elemento altamente poluente do meio ambiente, poderá proporcionar uma sensível economia no produto final. Considerando a sua substituição total ou parcial por elementos naturais e de uso tradicional dentro da construção civil, não esquecendo que um dos principais aspectos e de grande relevância na utilização desse material é o ecológico.

No intuito de contribuir com soluções alternativas para a destinação dos resíduos de pneus provenientes das indústrias recauchutadoras com o seu uso dentro da indústria da construção civil, com vistas à economia e melhoria de alguma das propriedades nos blocos de concreto vazado para alvenaria de vedação é que o presente trabalho buscou focar o uso desse resíduo, como forma de adição, em traços de concreto que fornecessem um melhor comportamento com relação a sua resistência mecânica com vistas a sua utilização futura.

\section{OBJETIVO}

O presente trabalho foi desenvolvido para identificar os efeitos do teor de borracha e a quantidade de água na resistência à compressão. Dessa forma, variando os parâmetros definidos acima, buscou-se o traço que conduzisse a maior resistência à compressão, associado à umidade ótima da mistura. Para o estudo foram produzidos 180 corpos-deprova de $10 \times 20 \mathrm{~cm}$, substituindo-se parte do agregado miúdo pelo resíduo de pneu reciclado, tornando-os mais leves e com a resistência esperada. A redução na massa específica devido a presença desse novo material na composição da mistura é de fundamental importância com relação a redução das cargas permanentes atuantes na estrutura, acarretando um economia final na estrutura (estrutura menos robusta).

\section{METODOLOGIA}

Para o desenvolvimento da pesquisa, buscou-se, inicialmente, a coleta do resíduo nas indústrias recauchutadoras de pneus na Ribeira-Natal/RN.

Os resíduos de pneus são obtidos através de processo mecânico que se utiliza de equipamento denominado "raspadeira" composto de cilindros dotados de ranhuras que provocam o desgaste na sua superfície de rodagem, por raspagem, nos pneus usados, para posterior colocação de uma nova capa de bandagem. 
O único tratamento dispensado a este resíduo foi a exclusão de partículas que tivessem diâmetros superiores a 9,5mm, adotando-se para isso o peneiramento. Constatou-se que as partículas maiores que 9,5mm de diâmetro representaram aproximadamente $1 \%$ da amostra total coletada (figura 1).

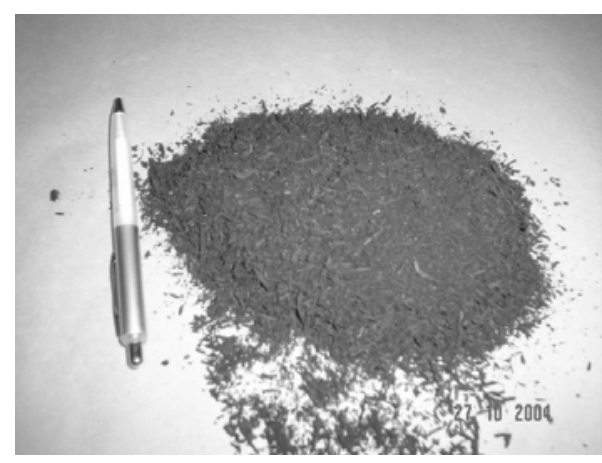

Figura 1 - Resíduo de pneu reciclado.

Os demais materiais, incluindo-se o resíduo de pneu, foram caracterizados através dos ensaios de granulometria, massa específica e massa unitária. Os agregados naturais utilizados foram os encontrados na região de Natal-RN. O cimento utilizado foi o CP II-Z 32.

Após a caracterização física dos materiais, optou-se por utilizar três traços para blocos de concreto proposto por ANDOFALTO (2002) na sua pesquisa, denominados de traços de referência com as seguintes nomenclaturas: T-1, T-2 e T-3. Inicialmente, moldaram-se corpos de prova com os respectivos traços de referência, variando para cada um deles o

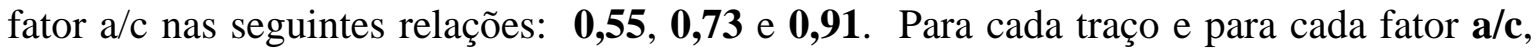
foram moldados 5 corpos-de-prova rompidos aos 28 dias de idade.

Após a moldagem dos traços de referência, variou-se a quantidade de resíduo de borracha em substituição parcial ao agregado graúdo, nas percentagens de 5, 10 e 15\%. Também foram moldados 5 corpos-de-prova para cada novo traço com borracha, agora denominados TR-1, TR-2 e TR-3, para verificação da resistência à compressão aos 28 dias.

\section{Caracterização dos Materiais}

A caracterização dos agregados naturais e da borracha, com relação a sua granulometria, foi baseada na NBR-7217:1987, que preconiza a utilização das peneiras da série normal e intermediária. Na tabela I estão indicadas as porcentagens retidas acumuladas de cada agregado.

Para determinação da massa específica da borracha, utilizou-se o Método do Picnômetro com substituição da água pelo Xilol, cuja densidade é menor que a água $\left(0,71 \mathrm{~g} / \mathrm{cm}^{3}\right)$. Todos os ensaios foram de acordo com as normas específicas da ABNT. A massa específica e massa unitária estão indicadas na tabela II. 
Tabela I - Porcentagem retida acumulada nas peneiras da série normal e intermediária.

\begin{tabular}{|c|r|r|r|}
\hline \hline $\begin{array}{c}\text { Peneiras } \\
(\mathrm{mm})\end{array}$ & Areia & Pedrisco & Borracha \\
\hline \hline 9,5 & & 6,1 & 0,1 \\
\hline 6,3 & 2,1 & 59,0 & 0,9 \\
\hline 4,8 & 2,8 & 88,3 & 2,1 \\
\hline 2,4 & 9,7 & 98,9 & 17,6 \\
\hline 1,2 & 38,3 & 99,1 & 59,5 \\
\hline 0,6 & 74,3 & 99,2 & 85,6 \\
\hline 0,3 & 91,1 & 99,3 & 96,4 \\
\hline 0,15 & 99,4 & 99,6 & 99,6 \\
\hline \hline
\end{tabular}

Tabela II - Caracterização física dos materiais.

\begin{tabular}{|c|c|c|c|c|}
\hline \hline & Areia & Pedrisco & Borracha & Cimento \\
\hline \hline $\begin{array}{c}\text { M.U. } \\
\left(\mathrm{kg} / \mathrm{dm}^{3}\right)\end{array}$ & 1,42 & 1,40 & 0,41 & 1,07 \\
\hline $\begin{array}{c}\text { M.E. } \\
\left(\mathrm{kg} / \mathrm{dm}^{3}\right)\end{array}$ & 2,59 & 2,59 & 1,61 & 3,02 \\
\hline \hline
\end{tabular}

\section{Produção dos corpos-de-prova de concreto}

Os corpos-de-prova de dimensões $10 \times 20 \mathrm{~cm}$ foram moldados com três traços diferentes, definidos como T1, T2 e T3. Entre os traços variaram o fator água/cimento e o percentual de resíduo de pneu reciclado (5\%, 10\% e 15\%). Os traços sem os resíduos de borracha serviram como traços de referência para análise final dos resultados. A mistura e homogeneização dos materiais ocorreram de forma mecânica usando-se uma betoneira com capacidade de 120l. Ao todo, foram moldados e ensaiados, para verificação da resistência à compressão, 180 corpos-de-prova. A moldagem de todos os corpos de prova para todos os traços analisados deu-se em acordo com a norma NBR-5738. Os traços em massa, fatores água/cimento, e percentagens do resíduo de pneu na mistura e quantidade de corpos-de-prova moldados, encontram-se indicados no tabela III.

\section{Cura dos Corpos-de-Prova}

Neste estudo, o processo de cura foi feito no laboratório do CEFET-RN nas condições ambientes de temperatura, fazendo a manutenção da umidade por meio de aspersão de água, excluindo-se o sábado e domingo, até a véspera do seu rompimento. Tal metodologia foi adotada no intuito de aproximar as mesmas condições de cura realizadas nas fábricas de blocos de concreto. 
Tabela III - Identificação dos traços sem e com borracha.

\begin{tabular}{|c|c|c|c|c|c|}
\hline \multicolumn{3}{|c|}{$\begin{array}{l}\text { TRAÇOS DE REFERÊNCIA } \\
\text { (SEM BORRACHA) }\end{array}$} & \multicolumn{3}{|c|}{ TRAÇOS COM BORRACHA } \\
\hline TRAÇO & Fator a/c & $\mathrm{CP}$ & TRAÇO & Fator a/c & $\mathrm{CP}$. \\
\hline \multirow{9}{*}{$\begin{array}{c}T-1 \\
1: 5,50: 3,50 \\
(1: 9)\end{array}$} & \multirow{3}{*}{$\mathrm{a} / \mathrm{c}=0,55$} & \multirow{3}{*}{5} & \multirow{3}{*}{$\begin{array}{c}\text { TR-1 } \\
1: 5,50 \\
: 3,33: 0,175 \\
(5 \%) \\
\end{array}$} & $\mathrm{a} / \mathrm{c}=0,55$ & 5 \\
\hline & & & & $\mathrm{a} / \mathrm{c}=0,73$ & 5 \\
\hline & & & & $\mathrm{a} / \mathrm{c}=0,91$ & 5 \\
\hline & \multirow{3}{*}{$\mathrm{a} / \mathrm{c}=0,73$} & \multirow{3}{*}{5} & \multirow{3}{*}{$\begin{array}{c}\text { TR-1 } \\
1: 5,50: 3,15: \\
0,35(10 \%)\end{array}$} & $\mathrm{a} / \mathrm{c}=0,55$ & 5 \\
\hline & & & & $\mathrm{a} / \mathrm{c}=0,73$ & 5 \\
\hline & & & & $\mathrm{a} / \mathrm{c}=0,91$ & 5 \\
\hline & \multirow{3}{*}{$\mathrm{a} / \mathrm{c}=0,91$} & \multirow{3}{*}{5} & \multirow{3}{*}{$\begin{array}{c}\text { TR-1 } \\
1: 5,50: 2,98: \\
0,53 \\
(15 \%) \\
\end{array}$} & $\mathrm{a} / \mathrm{c}=0,55$ & 5 \\
\hline & & & & $\mathrm{a} / \mathrm{c}=0,73$ & 5 \\
\hline & & & & $\mathrm{a} / \mathrm{c}=0,91$ & 5 \\
\hline \multirow{9}{*}{$\begin{array}{c}\text { T-2 } \\
1: 4,75: 3,00 \\
(1: 7,75)\end{array}$} & \multirow{3}{*}{$\mathrm{a} / \mathrm{c}=0,55$} & \multirow{3}{*}{5} & \multirow{3}{*}{$\begin{array}{c}\text { TR-2 } \\
1: 4,75: 2,85: \\
0,15 \\
(5 \%)\end{array}$} & $\mathrm{a} / \mathrm{c}=0,55$ & 5 \\
\hline & & & & $\mathrm{a} / \mathrm{c}=0,73$ & 5 \\
\hline & & & & $\mathrm{a} / \mathrm{c}=0,91$ & 5 \\
\hline & \multirow{3}{*}{$\mathrm{a} / \mathrm{c}=0,73$} & \multirow{3}{*}{5} & \multirow{3}{*}{$\begin{array}{c}\text { TR-2 } \\
1: 4,75: 2,70: \\
0,30 \\
(10 \%)\end{array}$} & $\mathrm{a} / \mathrm{c}=0,55$ & 5 \\
\hline & & & & $\mathrm{a} / \mathrm{c}=0,73$ & 5 \\
\hline & & & & $\mathrm{a} / \mathrm{c}=0,91$ & 5 \\
\hline & \multirow{3}{*}{$\mathrm{a} / \mathrm{c}=0,91$} & \multirow{3}{*}{5} & \multirow{3}{*}{$\begin{array}{c}\text { TR-2 } \\
1: 4,75: 2,55: \\
0,45 \\
(15 \%) \\
\end{array}$} & $\mathrm{a} / \mathrm{c}=0,55$ & 5 \\
\hline & & & & $\mathrm{a} / \mathrm{c}=0,73$ & 5 \\
\hline & & & & $\mathrm{a} / \mathrm{c}=0,91$ & 5 \\
\hline \multirow{9}{*}{$\begin{array}{c}\text { T-3 } \\
1: 6,75: 4,25 \\
(1: 11)\end{array}$} & \multirow{3}{*}{$\mathrm{a} / \mathrm{c}=0,55$} & \multirow{3}{*}{5} & \multirow{3}{*}{$\begin{array}{c}\text { TR-3 } \\
1: 6,75: 4,04: \\
0,21 \\
(5 \%)\end{array}$} & $\mathrm{a} / \mathrm{c}=0,55$ & 5 \\
\hline & & & & $\mathrm{a} / \mathrm{c}=0,73$ & 5 \\
\hline & & & & $\mathrm{a} / \mathrm{c}=0,91$ & 5 \\
\hline & \multirow{3}{*}{$\mathrm{a} / \mathrm{c}=0,73$} & \multirow{3}{*}{5} & \multirow{3}{*}{$\begin{array}{c}\text { TR-3 } \\
1: 6,75: 3,83: \\
0,43 \\
(10 \%)\end{array}$} & $\mathrm{a} / \mathrm{c}=0,55$ & 5 \\
\hline & & & & $\mathrm{a} / \mathrm{c}=0,73$ & 5 \\
\hline & & & & $\mathrm{a} / \mathrm{c}=0,91$ & 5 \\
\hline & \multirow{3}{*}{$\mathrm{a} / \mathrm{c}=0,91$} & \multirow{3}{*}{5} & \multirow{3}{*}{$\begin{array}{c}\text { TR-3 } \\
1: 6,75: 3,61: \\
0,64 \\
(15 \%) \\
\end{array}$} & $\mathrm{a} / \mathrm{c}=0,55$ & 5 \\
\hline & & & & $\mathrm{a} / \mathrm{c}=0,73$ & 5 \\
\hline & & & & $\mathrm{a} / \mathrm{c}=0,91$ & 5 \\
\hline
\end{tabular}

\section{Capeamento dos Corpos-de-prova}

Para o capeamento dos corpos de prova foi utilizado o método usado pelo laboratório da fabrica de cimento ITAPETINGA do grupo João Santos, situado no município de Mossoró, com resultados bastante satisfatórios. Tal método consistiu na substituição do capeamento tradicional que é realizado com enxofre, por dois pedaços de couro cru de $3 \mathrm{~mm}$ de 
espessura, posicionados nas faces superior e inferior do corpo-de-prova quando na aplicação do carregamento (figura 2).

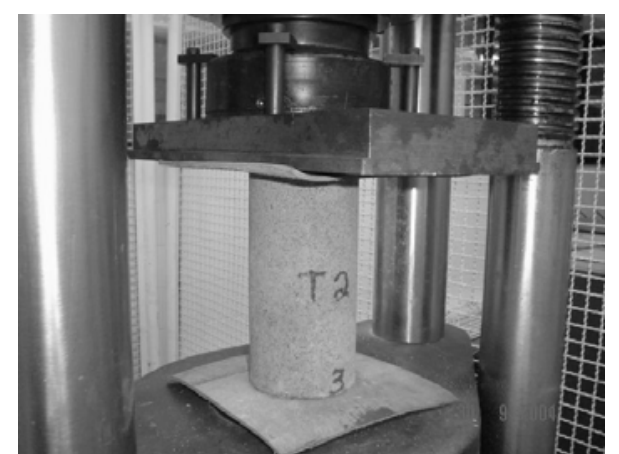

Figura 2 - Detalhe do capeamento dos corpos-de-prova e prensa de rompimento.

\section{Ensaios de Resistência à Compressão}

A determinação da resistência à compressão foi executada segundo a NBR - 5739 depois de decorridos 28 dias da moldagem dos corpos-de-prova. A prensa para rompimento dos corpos-de-prova foi do tipo universal, marca EMIC DL2000, com célula de carga (TRD 30) acoplada a mesma e aquisição de dados via computador (figura 2), através do programa MTest versão 2.00. Todos os corpos-de-prova foram ensaiados no laboratório do curso de Engenharia Civil da Universidade Potiguar.

\section{RESULTADOS E ANÁLISE DOS ENSAIOS}

Um dos principais focos, quando na análise do concreto endurecido, é a propriedade mecânica relativa a sua resistência à compressão, que serve como parâmetro direto de avaliação da qualidade desse material e por isso de suma importância quando na apresentação dos resultados. Na figura 3 apresentam-se as resistências à compressão dos traços de referência (sem resíduo). A maior resistência à compressão foi obtida no traço T2 para o fator $\mathrm{a} / \mathrm{c}=0,91$.

Quando comparadas, isoladamente, as resistências à compressão dos traços de referência com os traços com adição de resíduo de pneu, observou-se um decréscimo na resistência em todos os traços, conforme apresentado nas figuras 4, 5 e 6. Partindo-se para análise dos traços entre si, o traço 2 (com e sem borracha) foi o que melhor sinalizou quais as proporções mais adequadas desses materiais para os traços em estudo, apresentando as maiores resistências à compressão, conforme pode ser analisado nas figuras 3, 4, 5 e 6.

Para os traços sem adição de borracha, as menores resistências foram observadas no traço 1 ( figura 3). O traço que apresentou a menor resistência à compressão, quando com adições de borracha, foi o traço 3 (figuras 4,5 e 6), independente do fator a/c. 
TRAÇOS SEM BORRACHA

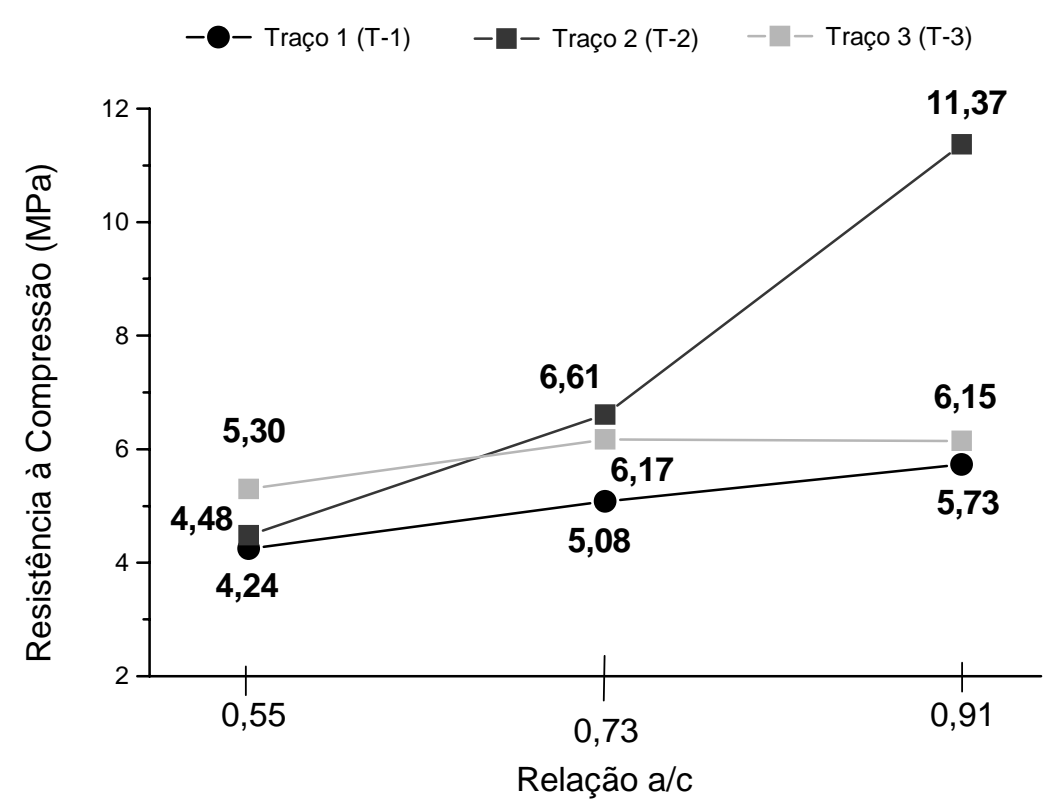

Figura 3 - Traços de Referência (sem borracha).

TRAÇOS COM 5\% DE BORRACHA

-0 - Traço 1 (TR-1 5\%) - - - - Traço 2 (TR-2 5\%) - - - Traço 3 (TR-3 5\%)

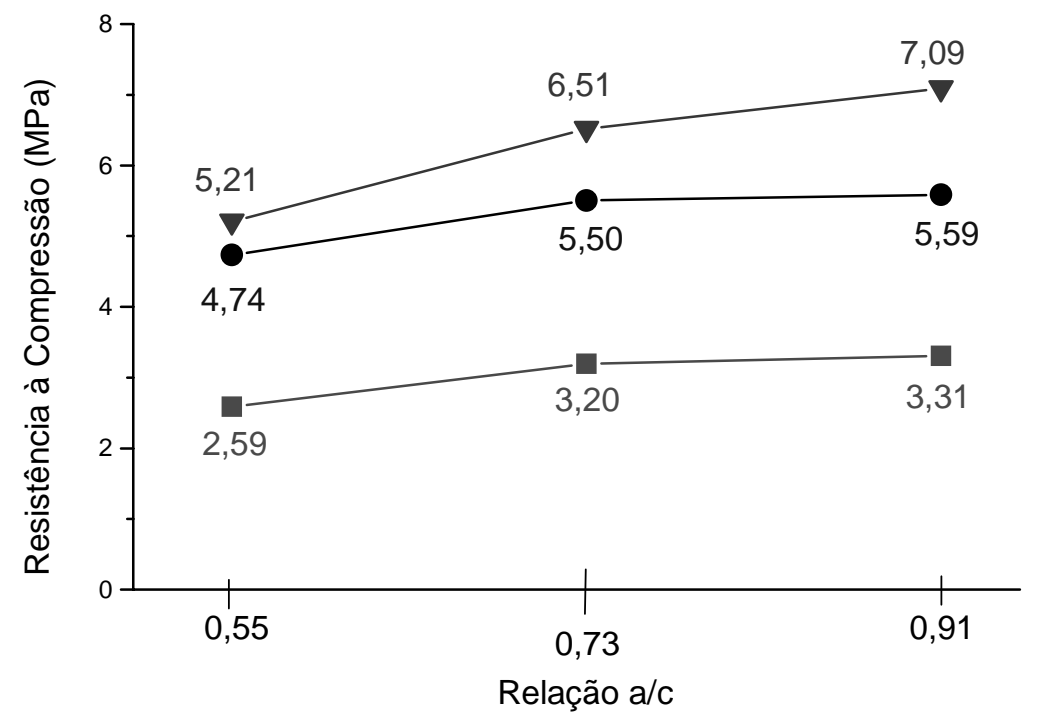

Figura 4 - Traços com 5\% de borracha. 
TRAÇOS COM 10\% DE BORRACHA

-0 - Traço 1 (TR-1 10\%) - - - Traço 2 (TR-2 10\%) - - - Traço 3 (TR-3 10\%)

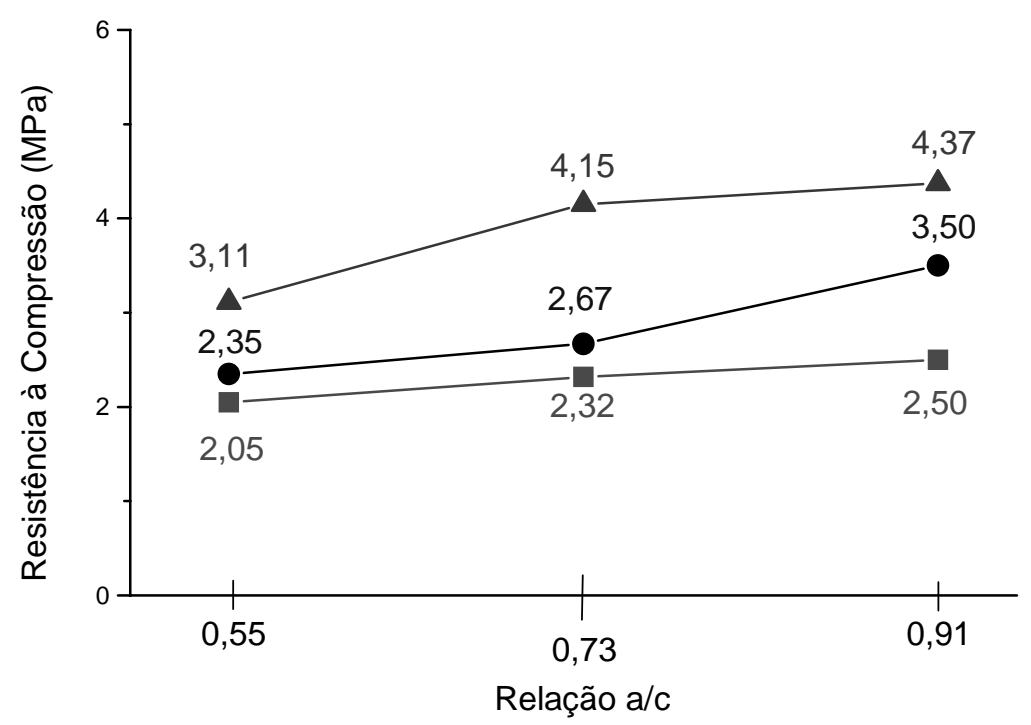

Figura 5 - Traços com 10\% de borracha.

TRAÇOS COM 15\% DE BORRACHA

-0 - Traço 1 (TR-1 15\%) - - $\mathbf{-}$ - Traço 2 (TR-2 15\%) - - - Traço 3 (TR-3 15\%)

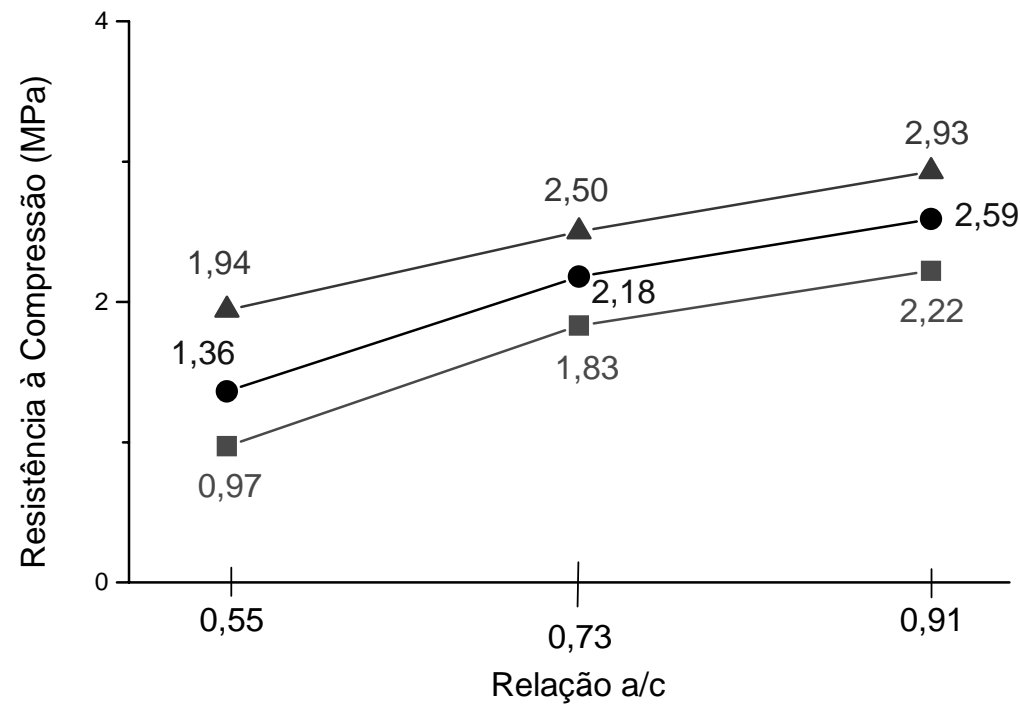

Figura 6 - Traços com 15\% de borracha. 


\section{CONCLUSÕES}

Com base nas análises das figuras apresentadas podemos concluir que:

- Os procedimentos investigatórios utilizados na pesquisa na busca do melhor traço, com relação ao comportamento mecânico (resistência à compressão) da mistura, mostrou-se satisfatório às analises desejadas;

- O traço que melhor apresentou resultados de resistência, sem a presença do resíduo de pneu, foi o traço T-2 para um fator água/cimento de 0,91(figura 3), chegando a uma resistência máxima de 11,37 $\mathrm{MPa}$. Fato este perfeitamente explicável devido o traço T-2 apresentar a menor quantidade de agregado em relação a quantidade de cimento (1: $7,75)$;

- Para os traços com adição de borracha para as três variações na percentagem de resíduo (5, 10 e 15\%) na mistura, o traço TR-2 foi o que apresentou a maior resistência à compressão (figuras 4, 5 e 6), com valores mais elevados para a relação a/c $=0,91$. Observa-se, assim como nos traços sem adição do resíduo, que a proporção entre agregados/cimento $(1: 7,75)$ permanece constante, tanto para o traço T-2 como para os traços 2 com adição de resíduo de pneu, sendo válida a explicação do item anterior;

- O traço que apresentou o maior valor de resistência à compressão foi o TR-2 (a/c = 0,91 ) com $5 \%$ de resíduo de borracha na sua composição chegando ao valor máximo de 7,09 MPa;

- Vale ressaltar que o resíduo de pneu exerceu uma influência significativa na diminuição da resistência à compressão em todos os traços com percentuais de $10 \%$ e 15\%. Nos estudos de TOPÇU (1997), recomenda-se quantidades de até 35\% de resíduo de borracha sem afetar significativamente a sua resistência mecânica, desde que o diâmetro das partículas do resíduo não ultrapasse $1 \mathrm{~mm}$, fato este que não podemos tomar como referência para a nossa pesquisa, uma vez que os diâmetros das partículas adicionadas aos nossos traços variaram de 0,15 a 9,5mm;

- Como não podemos fazer uma relação direta da resistência obtida nos corpos-deprova com a resistência em blocos de concreto, mesmo mantendo-se a proporção dos materiais, é que a pesquisa continuará sendo desenvolvida com a utilização dos traços estudados na confecção de blocos de concreto.

\section{REFERÊNCIAS BIBLIOGRÁFICAS}

ABNT - Associação Brasileira de Normas Técnicas. NBR-7251. Agregado Determinação da Massa Específica de Agregados por Meio do Frasco de Chapman. Rio de Janeiro, Brasil, 1987.

ABNT - Associação Brasileira de Normas Técnicas. NBR-5739. Concreto - Ensaio de compressão de corpos-de-prova cilíndricos. Rio de Janeiro, Brasil, 1994. 
ABNT - Associação Brasileira de Normas Técnicas. NBR NM225 - Critérios mínimos de seleção de pneus para reforma e reparação. Inspeção e identificação. Rio de Janeiro, Brasil, 2000.

Andofalto, R. P., Camacho, J. S. e Maurício, R. M. Blocos de Concreto: A busca de um Traço Otimizado. Unesp, Ilha Solteira, São Paulo, Brasil, 2002.

Sousa, J. G. G., Bauer, E. e Sposto, R. M. Blocos de Concreto Produzidos com Agregados Provenientes da Reciclagem de Resíduos Gerados pela Construção Civil. IX Encontro Nacional de Tecnologia do Ambiente Construído, Paraná, Brasil, 2002.

Topçu, I. B., e Avcular, N. Analysis of Rubberized Concrete as a Composite Material. Cemente and Concrete Reseach. USA, 1997.

Soares, R. C., Costa Neto, L. X. da, Mendes, J. U. L. e Marinho, G. S. Uso de resíduo de pneu como agregado de cimento portland. I Simpósio de Conforto Térmico da UFPI, Teresina, Piauí, Brasil, 2003. 